\title{
Growth of anisotropic gold nanostructures on conducting glass surfaces
}

\author{
P R SAJANLAL and T PRADEEP* \\ DST Unit on Nanoscience (DST UNS), Department of Chemistry and Sophisticated \\ Analytical Instrument Facility, Indian Institute of Technology Madras, Chennai 600036 \\ e-mail: pradeep@iitm.ac.in
}

\begin{abstract}
In this paper, we describe a method for the growth of gold nanowires and nanoplates starting from a bilayer array of gold seeds, anchored on electrically conducting indium tin oxide (ITO) substrates. This is based on a seed-mediated growth approach, where the nanoparticles attached on the substrate through molecular linkages are converted to nanowires and nanoplates at certain cetyltrimethylammonium bromide (CTAB) concentration. Our modified approach can be used to make nanowires of several tens of micrometers length at a lower CTAB concentration of $0 \cdot 1 \mathrm{M}$. The length of the nanowires can be varied by adjusting the time of the reaction. As the concentration of CTAB was increased to $0 \cdot 25 \mathrm{M}$, the nanoparticles got converted to nanoplates. These Au nanoplates are (111) oriented and are aligned parallel to the substrate.
\end{abstract}

Keywords. Gold; ITO; nanowires; nanoplates; seed-mediated growth.

\section{Introduction}

Synthesis of metal nanoparticles of diverse shape and size has become a major area of research due to their geometry-dependent properties and potential applications in chemical ${ }^{1} /$ optical sensing, ${ }^{2}$ nanoelectronics, ${ }^{3}$ surface enhanced Raman scattering (SERS), ${ }^{4}$ metal enhanced fluorescence ${ }^{5}$ and catalysis. ${ }^{6}$ Heterostructure assembly of such hybrid wires exhibits exceptional electron transfer properties. ${ }^{7}$ Several synthetic approaches such as photochemical, ${ }^{8}$ biological, ${ }^{9}$ templated, ${ }^{10}$ electrochemical ${ }^{11}$ and surfactant-based seed-mediated ${ }^{12}$ growth methods have been reported for making anisotropic nanostructures of gold and silver. In order to fabricate functional materials and devices, it is necessary to have an assembly of these nanoparticles onto solid planar substrates. For this, one can use sophisticated methods such as nanosphere lithography. ${ }^{13}$ Several other methods are also employed for the fabrication of one-dimensional (1D) and two-dimensional (2D) nanostructures on planar surfaces such as seed mediation, ${ }^{14}$ immobilization of nanostructures onto self-assembled monolayers (SAMs) via covalent interactions, ${ }^{15}$ growth of anisotropic nanostructures along the direction of gas flow, ${ }^{16}$ electrochemical deposi-

\footnotetext{
*For correspondence
}

tion, ${ }^{17}$ galvanic displacement reactions, ${ }^{18}$ sputter deposition and thermal vapor deposition. ${ }^{19}$ We have explored several possibilities of immobilized metal nanoparticle assemblies on planar surfaces in order to make sensors and devices. ${ }^{20,21}$ At present, the fabrication of uniform anisotropic metal nanostructures like Au plates and wires with high yield and welldefined morphologies is still a challenge.

Our research group is actively engaged in the synthesis, characterization and application of various anisotropic metal nanostructures. We developed a methodology to fabricate aligned array of equilateral gold nanotriangles on ITO substrate via a potential assisted seed-mediated approach. ${ }^{22}$ This substrate showed an intense near infrared (NIR) absorption and strong surface enhanced Raman activity. We have recently reported the cupric ion assisted shape transformation of AuNRs into spherical AuNPs. ${ }^{23}$ The intermediate structures formed during this shape transformation could be arrested at any stage to produce nanoparticles of preferred shape. Here, selective etching of the tips of the nanorods occurs at higher concentration of CTAB and subsequent shape transformation is driven by the surface reconstruction of nanorods to produce more stable planes. A solution phase method for assembling gold nanorodcoated poly $(N$-isopropyl acrylamide) microgels into a hexagonal pattern was reported recently from our group. ${ }^{24}$ These patterns were made by growing the 
seed nanoparticle attached to the microgels into nanorods.

In this paper, we report a method for the growth of 1D and 2D nanostructures of gold such as nanowires and nanoplates on ITO surfaces by a seedmediated growth method. Several such seed-mediated processes have been reported in the recent past, especially for anisotropic nanostructures such as nanorods. The method reported here is an adaptation of the seed mediated growth of gold nanorods on mica surfaces reported by Taub et al. ${ }^{25}$ Our method enables the control of size and shape of the nanostructures on ITO by varying the experimental parameters such as the growth time, concentration of surfactant and temperature of the reaction.

\section{Experimental}

\subsection{Materials}

Tetrachloroauric acid trihydrate $\left(\mathrm{HAuCl}_{4} \cdot 3 \mathrm{H}_{2} \mathrm{O}\right)$, CTAB, sodium citrate and ascorbic acid (AA) were purchased from $\mathrm{CDH}$, India. Sodium borohydride $\left(\mathrm{NaBH}_{4}\right), 1,9$-nonanedithiol (NDT) and aminopropytrimethoxysilane (APTMS) were purchased from Aldrich. ITO with a resistivity of $70 \Omega / \mathrm{cm}$ was used throughout this work. All chemicals were used as such without further purification. Triply distilled water was used throughout the experiments.

Scheme 1 shows the experimental procedure employed for making nanowires and nanoplates on ITO substrate.

\subsection{Preparation of ITO substrates}

The ITO glass slides of dimensions $1 \mathrm{~cm} \times 2 \mathrm{~cm}$ were sonicated with a mild detergent solution, ethanol and finally with pure water (in the order mentioned). They were then soaked in a $10 \% \mathrm{HCl}$ solution for activation, washed with water, and dried under a stream of nitrogen. These plates were annealed at $450^{\circ} \mathrm{C}$ for $7 \mathrm{~h}$ and cooled in a desiccator. The glass slides were dipped in $30 \mathrm{mM}$ APTMS solution in methanol for $1 \mathrm{~h}$. Afterwards, they were washed with methanol and water in sequence and kept at $100^{\circ} \mathrm{C}$ for $2 \mathrm{~h}$.

\subsection{Preparation of gold seed}

4 to $8 \mathrm{~nm}$ diameter AuNP seeds were synthesized by taking $37 \mathrm{~mL}$ of water in a beaker and $1 \mathrm{~mL}, 0.01 \mathrm{M}$ $\mathrm{HAuCl}_{4} \cdot 3 \mathrm{H}_{2} \mathrm{O}$ and $1 \mathrm{~mL}, 0.01 \mathrm{M}$ aqueous trisodium-

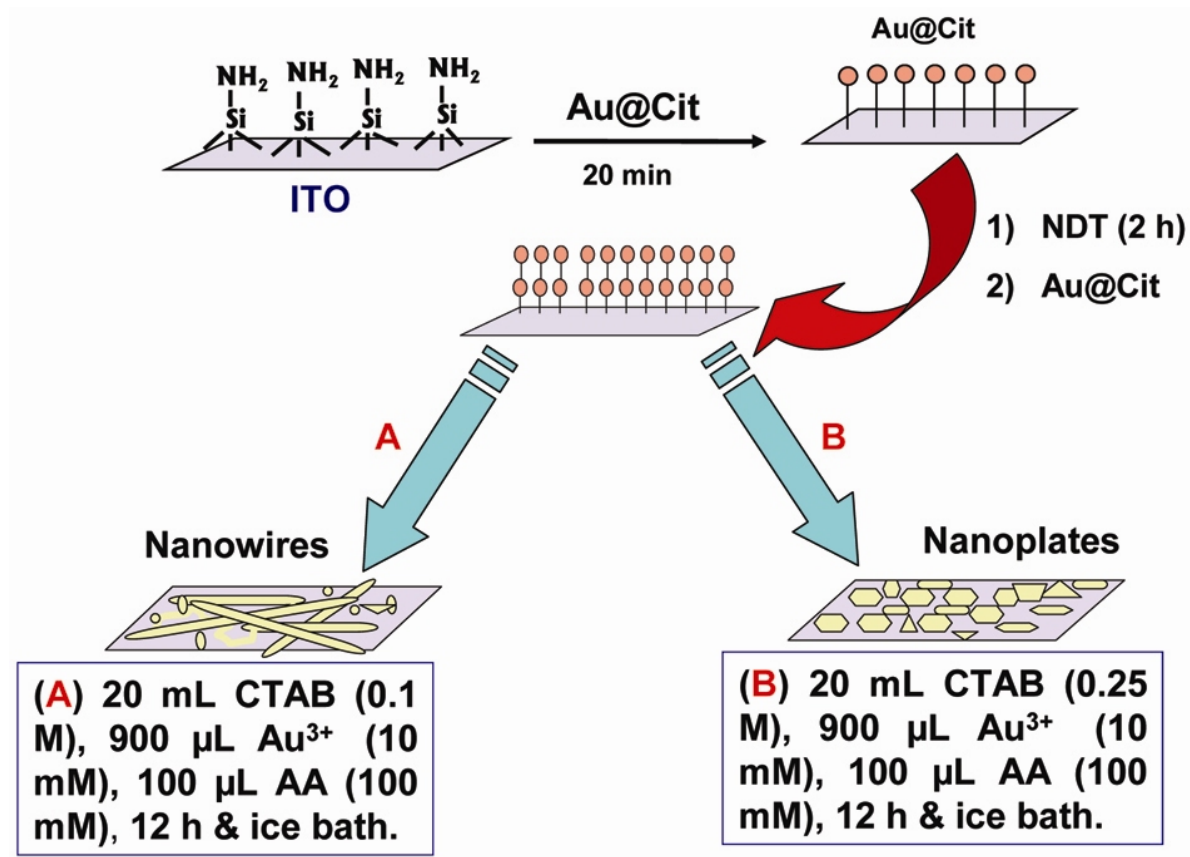

Scheme 1. Procedure for the growth of gold nanoplates and nanowires on ITO substrates. 

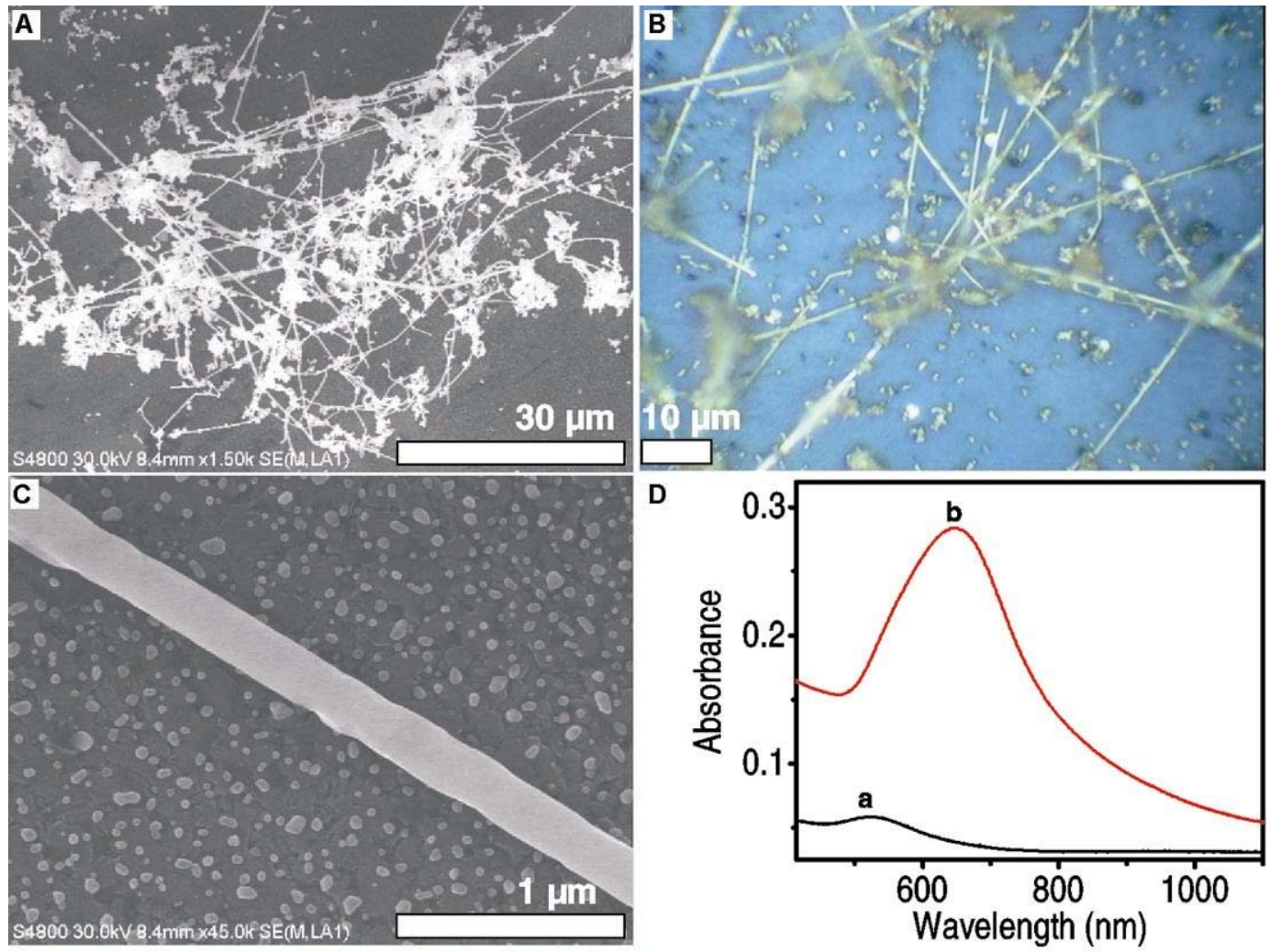

Figure 1. (A) SEM image of gold nanowires formed on ITO after $12 \mathrm{~h}$ of the reaction; (B) optical image of the nanowires formed after $12 \mathrm{~h}$ of reaction; (C) SEM image of an enlarged portion of a single gold nanowire and (D) UV-vis spectra of the nanoparticle coated ITO (a) and the nanowires formed (b).

citrate solution was added to it. Then $1 \mathrm{~mL}$ of ice cold solution of $0 \cdot 1 \mathrm{M}$ aqueous $\mathrm{NaBH}_{4}$ was added and the solution was stirred for $5 \mathrm{~min}$.

\subsection{Growth of gold nanowires on ITO substrates}

The APTMS coated ITO substrate was immersed in the seed solution for a period of $20 \mathrm{~min}$. The substrate was washed with water and dried. This substrate was then dipped in a $30 \mathrm{mM}$ solution of NDT in methanol for $2 \mathrm{~h}$. Subsequently, this substrate was dipped in the nanoparticle solution (20 $\mathrm{min})$ again, in order to get a bilayer assembly of nanoparticles.

The bilayer of seed coated ITO substrate was then immersed in $20 \mathrm{~mL}, 0.1 \mathrm{M} \mathrm{CTAB}$ solution for $3 \mathrm{~min}$. After this $900 \mu \mathrm{L}, 0.01 \mathrm{M} \mathrm{HAuCl}_{4} \cdot 3 \mathrm{H}_{2} \mathrm{O}$ was added followed by $100 \mu \mathrm{L}, 0 \cdot 1 \mathrm{M}$ ascorbic acid. This growth vessel was kept inside an ice bath throughout the process and allowed to stand undisturbed for $12 \mathrm{~h}$. The nanowire formation was evident by the colour change of the ITO substrate from colourless to sandlike appearance. In order to get nanowires of various lengths, we changed the reaction time. It was noted that as the reaction time increases, the length of the nanowires was increased. After the completion of the growth, the substrate was taken out, washed using distilled water in order to remove the excess CTAB and was characterized.

\subsection{Growth of gold nanoplates on ITO substrates}

The bilayer of seed coated ITO substrate was immersed in $20 \mathrm{~mL}, 0.25 \mathrm{M} \mathrm{CTAB}$ solution for $3 \mathrm{~min}$. After this, $900 \mu \mathrm{L}, 0.01 \mathrm{M} \mathrm{HAuCl}_{4} \cdot 3 \mathrm{H}_{2} \mathrm{O}$ was added followed by $100 \mu \mathrm{L}, 0 \cdot 1 \mathrm{M}$ ascorbic acid. This growth vessel was kept inside an ice bath throughout the process and allowed to stand undisturbed for $12 \mathrm{~h}$. The nanoplate formation was evident by the colour change of the ITO substrate from colourless to pale pink.

\subsection{Instrumentation}

Scanning electron microscopic (SEM) and energy dispersive analysis of X-rays (EDAX) studies were 

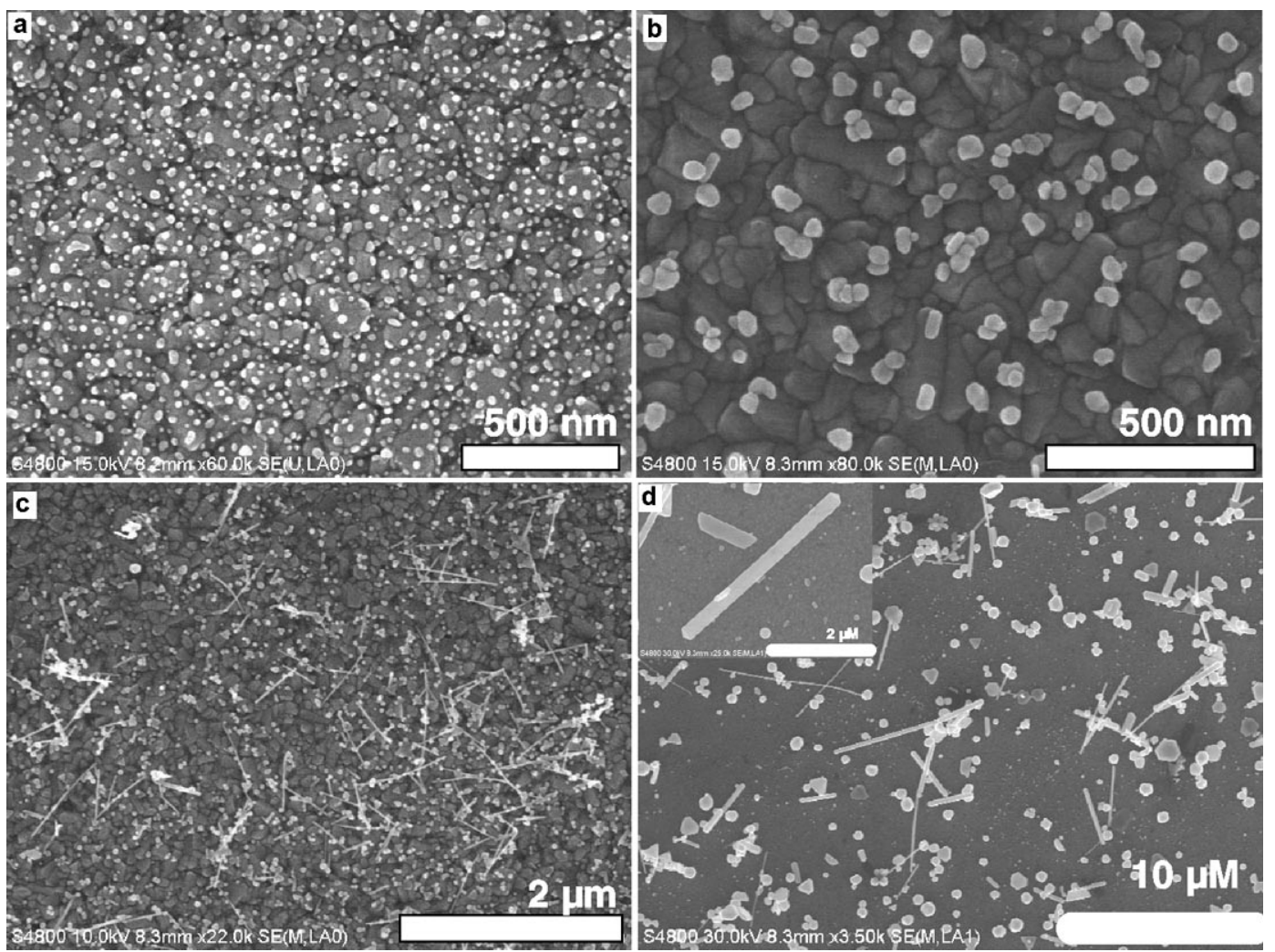

Figure 2. SEM images showing the gold nanowires formed at different time intervals (a) $15 \mathrm{~min}$, (b) $30 \mathrm{~min}$, (c) $4 \mathrm{~h}$ and (d) $8 \mathrm{~h}$. Inset shows the SEM image of a single nanowire formed after $8 \mathrm{~h}$.

done in a HITACHI S-4800 FE-SEM. UV-vis spectra were measured using a Perkin-Elmer Lambda 25 spectrometer. Optical images were taken using a WiTec GmbH, Alpha-SNOM CRM 200 spectrometer. X-ray diffraction (XRD) data were collected with a Shimadzu XD-D 1 diffractometer using $\mathrm{CuK} \alpha$ radiation $(\lambda=1.54 \AA)$. The samples were scanned in the $2 \theta$ range of $10-90$ degrees.

\section{Results and discussion}

The gold nanowires and nanoplates were grown on ITO substrate via a modified seed-mediated growth method. The characterization of these nanostructures was done by optical absorption measurements, SEM and XRD. We succeeded to tune the length of nanowires ranging from few nanometres to several tens of micrometers. Figure 1A shows a large area SEM image of the gold nanowires formed after $12 \mathrm{~h}$ of reaction. It is clear from the image that a large number of nanowires were formed and most of the nanowires have more than $100 \mu \mathrm{m}$ length. These nanowires were also visible under the optical micro- scope. Figure 1B shows an optical microgram of the nanowires grown under similar conditions. Figure $1 \mathrm{C}$ shows an enlarged portion of a single nanowire. The diameter of these nanowires is in the range of $\sim 100 \mathrm{~nm}$. UV-vis spectra of nanoparticle-coated ITO and nanowires formed after $12 \mathrm{~h}$ of the reaction are shown in figure 1D. The UV-vis spectrum of the AuNPs coated ITO exhibited an absorption maximum at $520 \mathrm{~nm}$, which is due to the surface plasmon resonance (SPR) of spherical gold nanoparticles. After the reaction, the SPR band of nanoparticle coated ITO red shifted to $650 \mathrm{~nm}$ indicating the growth of nanowires. The background of the absorption spectrum slightly increased after the reaction indicating the formation of large anisotropic nanostructures.

The length of the nanowires can be tuned by varying the reaction time. As the time of reaction increases, the length of the nanowires was found to be increased. One of the advantages of our synthetic approach over the other seed-mediated growth methods reported earlier is that we can make nanowires of length larger than $100 \mu \mathrm{m}$. Such nanowires would be useful for making conductive components in 

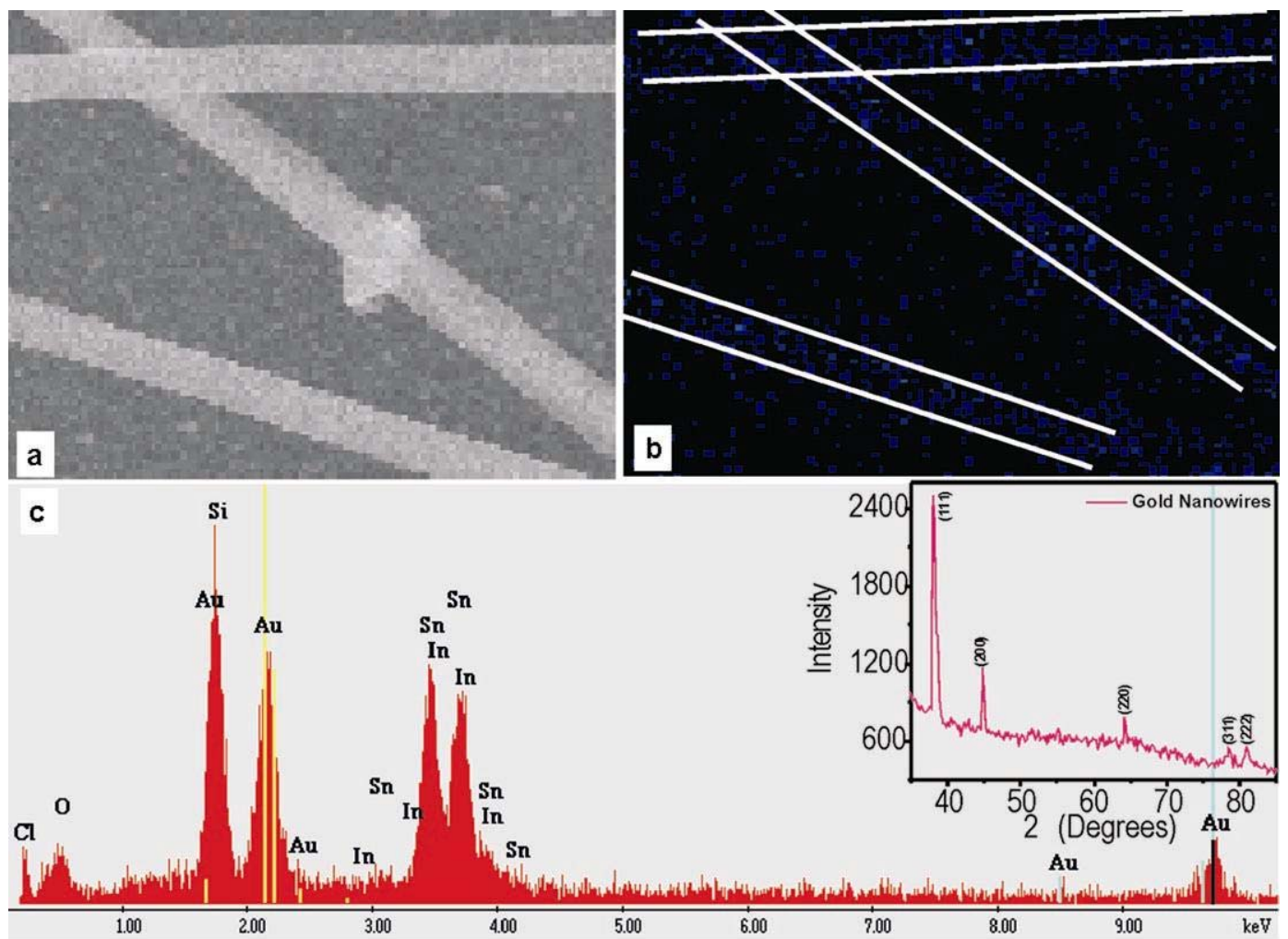

Figure 3. (a) SEM image of the nanowires formed on ITO after $12 \mathrm{~h}$; (b) EDAX mapping of Au nanowires shown in a and (c) EDAX spectrum of a gold nanowire. Inset of c shows an XRD pattern of the nanowires formed after $12 \mathrm{~h}$ of the reaction.

electronics. We can make nanowires of desirable length by stopping the growth at various stages of the reaction. Figures $2 a-d$ show the nanowires formed at various time intervals such as $15 \mathrm{~min}, 30 \mathrm{~min}, 4 \mathrm{~h}$ and $8 \mathrm{~h}$ of the reaction, respectively. After $30 \mathrm{~min}$ of the reaction, the nanoparticles grown on the substrate were smaller rods of $\sim 100 \mathrm{~nm}$ length. After $4 \mathrm{~h}$ of the reaction, the length of most of the nanowires were 1 to $2 \mu \mathrm{m}$. Inset of figure $2 \mathrm{~d}$ shows the SEM image of a single nanowire formed after $8 \mathrm{~h}$ of the reaction.

The nanowire growth happens from the multiply twinned seed nanoparticles by the passivation of certain crystallographic planes of the Au seed by the adsorption of CTAB. This results in the growth of anisotropic particles. The CTAB forms a bilayer which stabilizes and retards the growth along $\mathrm{Au}$ (110) face of the nanorods and promotes the growth at the tip. ${ }^{26}$ Low temperature enhances the growth rate. It is noticed that the bilayer of nanoparticles immobilized on the ITO results in the formation of much lengthy nanowires compared to a monolayer of nanoparticles. In the case of a monolayer of nanoparticles, the maximum length of the nanowires was found to be less than $5 \mu \mathrm{m}$. Figure 3 a shows a magnified SEM image of nanowires grown on ITO after $12 \mathrm{~h}$ of reaction and corresponding EDAX mapping of $\mathrm{Au}$ of the these nanowires is shown in figure $3 \mathrm{~b}$. An EDAX map and the spectrum taken from the nanowires confirm that these nanostructures are made of gold. This is further confirmed by XRD. Inset of figure $3 \mathrm{c}$ shows the XRD of the as prepared nanowires coated ITO substrate. The XRD patterns of the nanowires were assigned to the (111), (200), (220), (311) and (222) reflections of face-centered cubic gold.

In the seed-mediated growth approach, the concentration of the surfactant plays an important role in the morphology determination. Mirkin et $a l^{27}$ synthesized nanotriangles starting from $4 \mathrm{~nm} \mathrm{Au} @$ Citrate seed nanoparticles using a growth solution containing saturated CTAB. In order to grow nanoplates on the ITO substrate, we increased the concentration of CTAB and kept all other parameters constant. We used $0.25 \mathrm{M}$ of CTAB. In the case of nanowire growth, it was $0.1 \mathrm{M}$. A typical large area SEM image of the gold nanoplates grown on ITO substrate at this condition after $12 \mathrm{~h}$ of reaction is shown in figure 4A. These nanoplates have smooth 


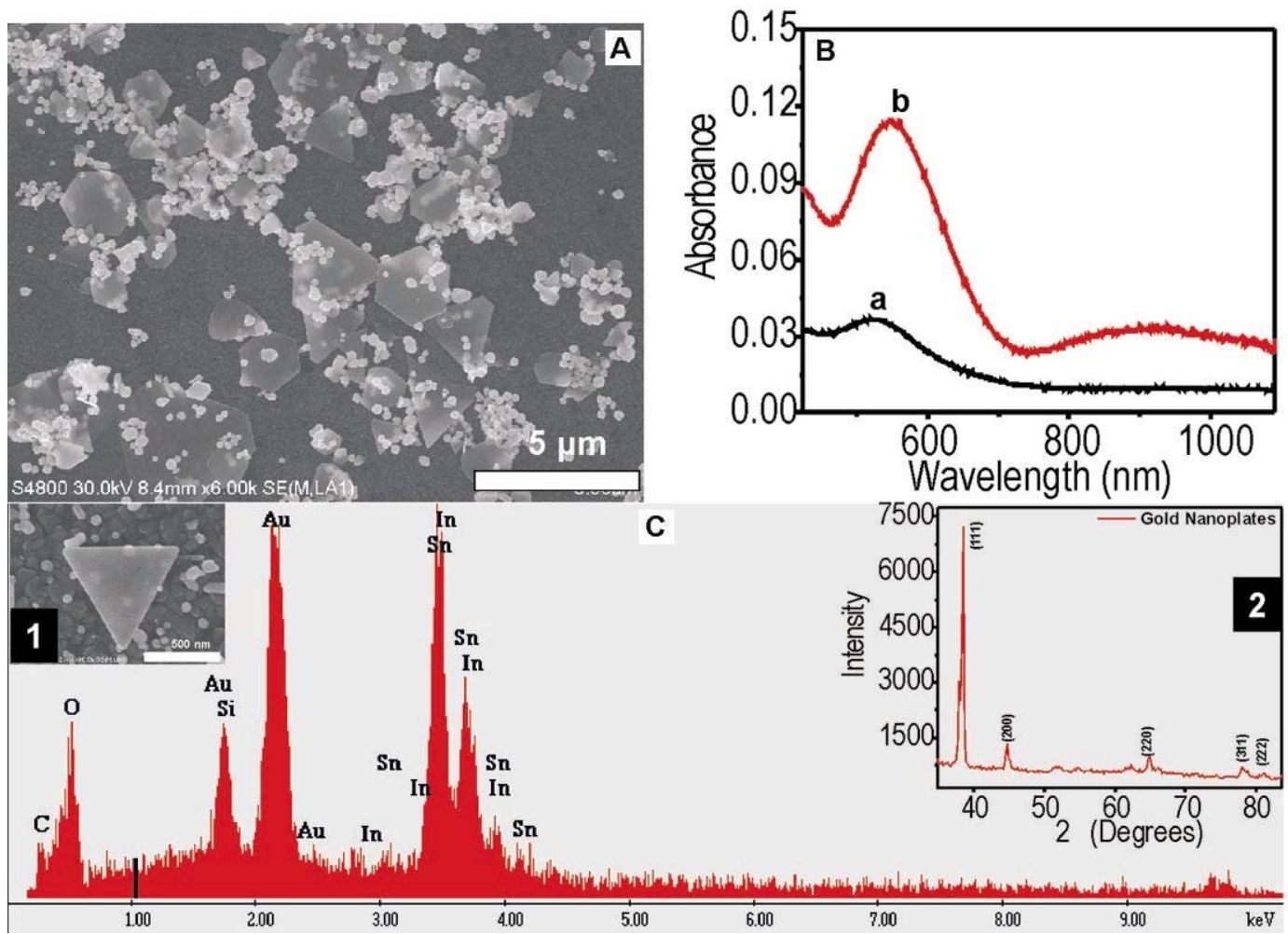

Figure 4. (A) SEM image of the gold nanoplates formed on ITO after $12 \mathrm{~h}$ of the reaction; (B) UV-vis spectra of (a) nanoparticle coated ITO and (b) nanoplates formed under the same condition and (C) EDAX spectrum taken from the nanoplate shown in inset 1. Inset 2 shows the XRD of the nanoplates formed under the same condition.

surfaces and have a thickness less than $50 \mathrm{~nm}$. With this procedure, nanoplates with an edge length from 1 to $3 \mu \mathrm{m}$ were formed on the ITO surface. These nanostructures contain hexagonal shaped nanoplates, nanotriangles and truncated triangular nanoplates. Figure 4B shows the absorption spectra of nanoparticle coated ITO and nanoplates formed after $12 \mathrm{~h}$. The absorption spectrum of the nanoparticle coated ITO shows a single absorption maximum at $520 \mathrm{~nm}$ and there was no absorption observed at longer wavelength. As the growth proceeds, the SPR of the nanoparticle centered at $520 \mathrm{~nm}$ red shifted to $550 \mathrm{~nm}$ and a broad absorption band appeared in the NIR region indicating the formation of gold nanoplates. The broad peak in the NIR region is attributed to in-plane SPR of nanoplates and that observed at $550 \mathrm{~nm}$ is due to their out of plane SPR. ${ }^{27}$

The intensity ratio of the (111) and (200) diffraction peaks in the XRD pattern (inset 2 of figure 4C) of nanoplates was higher than the bulk value. This result suggests that the $\mathrm{Au}$ nanoplates were rich in (111) planes and their (111) planes are preferentially oriented parallel to the surface of the ITO substrate. The EDAX spectrum (figure 4C) taken from a single nanoplate (inset 1) confirms that these nanoplates are made of gold. The dramatic change in the morphology is mainly attributed to the adsorption of CTAB on low index planes of gold. The growth solution contains $\mathrm{CTAB}$ in much higher concentration $(0.25 \mathrm{M})$ which suppresses the overall crystal growth through specific adsorption on certain planes. This favours the formation of (111) oriented nanoplates. Low temperature favours the slow growth of the nanoparticles into nanoplates. It was noted that the bilayer of nanoparticle also has some influence on the formation of nanoplates. At the same condition, monolayers of nanoparticle were grown into bigger nanoparticles of irregular geometry.

\section{Conclusion}

In conclusion, this paper describes a simple method to grow $\mathrm{Au}$ nanowires of length greater than $100 \mu \mathrm{m}$ and nanoplates of edge length $1-3 \mu \mathrm{m}$ on ITO sub- 
strates via a modified seed-mediated growth approach. These nanostructures were grown on an ITO substrate covered with a bilayer of Au nanoparticles. The length of the nanowire can be varied by adjusting the time of the reaction. It was found that the concentration of CTAB and temperature play an important roles in the morphology determination. The products were characterized by UV-vis spectroscopy, SEM, XRD and optical microscopy. These nanostructures could have promising applications in surface enhancement spectroscopic methods.

\section{Acknowledgements}

We thank the Department of Science and Technology, Government of India for constantly supporting our research program on nanomaterials. Prof Keisaku Kimura, Department of Material Science, University of Hyogo, Japan is thanked for allowing the use of his SEM. Dr A Sreekumaran Nair is thanked for assistance with the SEM measurements.

\section{References}

1. Haes A J, Hall W P, Chang L, Klein W L and Van Duyne R P 2004 Nano Lett. 41029

2. Haes A J and Van Duyne R P 2002 J. Am. Chem. Soc. 12410596

3. (a) Kovtyukhova N I, Martin B R, Mbindyo J K N, Smith P A, Razavi B, Mayer T S and Mallouk T E 2001 J. Phys. Chem. B105 8762; (b) Sun S H, Murray C B, Weller D, Folks L and Moser A 2000 Science 2871989

4. (a) Campion A and Kambhampati P 1998 Chem. Soc. Rev. 27 241; (b) Tian Z, Ren B and Wu D $2002 J$. Phys. Chem. B106 9463

5. Aslan K, Lakowicz J R and Geddes C D $2005 \mathrm{~J}$. Phys. Chem. B109 6247

6. Street S C, Xu C and Goodman D W 1997 Annu. Rev. Phys. Chem. 4843
7. Sharma J, Vivek J P, Vijayamohanan K P, Singh $P$ and Dharmadhikari C V 2006 Appl. Phys. Lett. 88 193103

8. (a) Maillard M, Huang P and Brus L 2003 Nano Lett. 3 1611; (b) Jin R, Cao Y, Mirkin C A, Kelly K L, Schatz G C and Zheng J G 2001 Science 2941901

9. Shankar S S, Rai A, Ankamwar B, Singh A, Ahmad A and Sastry M 2004 Nat. Mater. 3482

10. (a) Hong B H, Bae S C, Lee C W, Jeong S and Kim K S 2001 Science 294 348; (b) Zong R L, Zhou J, Li Q, Du B, Li B, Fu M, Qi X W, Li L T and Buddhudu S 2004 J. Phys. Chem. B108 16713; (c) Adhyapak P V, Karandikar P, Vijayamohanan K, Athawale A A and Chandwadkar A J 2004 Mater. Lett. 58168

11. Yu Y Y, Chang S S, Lee C L and Chris Wang C R 1997 J. Phys. Chem. B101 6661

12. Busbee B, Obare S and Murphy C J 2003 Adv. Mater. 15414

13. Haynes C L and Van Duyne R P 2003 Nano Lett. 3 939

14. Umar A A and Oyama M 2006 Cryst. Growth Des. 6818

15. Gole A, Orendorff C J and Murphy C J 2004 Langmuir 207117

16. Huang S, Cai X and Liu J 2003 J. Am. Chem. Soc. 1255636

17. (a) Notsu H, Kubo W, Shitanda I and Tatsuma T 2005 J. Mater. Chem. 15 1; (b) Tian Y, Liu H, Zhao $\mathrm{G}$ and Tatsuma T 2006 J. Phys. Chem. B110 23478

18. Wiederrecht G P and Sun Y 2007 Small 31964

19. Jia D and Goonewardene A 2006 Appl. Phys. Lett. 88 53105

20. Subramaniam C, Pradeep T and Chakrabarti J 2005 Phys. Rev. Lett. 95164501

21. Subramaniam C, Pradeep T and Chakrabarti J $2007 \mathrm{~J}$. Phys. Chem. C111 19103

22. Sajanlal P R and Pradeep T Adv. Mater. (in press)

23. Sreeprasad T S, Samal A K and Pradeep T 2007 Langmuir 239463

24. Rajeev Kumar V R, Samal A K, Sreeprasad T S and Pradeep T 2007 Langmuir 238667

25. Taub N, Krichevski O and Markovich G $2003 \mathrm{~J}$. Phys. Chem. B107 11579

26. Wei Z and Zamborini F P 2004 Langmuir 2011301

27. Millstone J E, Park S, Shuford K L, Qin L, Schatz G C and Mirkin C A 2005 J. Am. Chem. Soc. 1275312 